\title{
Enabling Remote Access to Personal Electronic Medical Records
}

James K. Archibald

james_archibald@byu.edu

Eric S. Hall

enrique.hall@gmail.com

Charles D. Knutson

knutson@cs.byu.edu

David K. Vawdrey

Follow this and additional works at: https://scholarsarchive.byu.edu/facpub

Part of the Computer Sciences Commons

\section{Original Publication Citation}

David K. Vawdrey, Eric S. Hall, Charles D. Knutson, James K Archibald. "Enabling Remote Access to Personal Electronic Medical Records. " IEEE Engineering in Medicine and Biology Magazine, vol. 22, no. 2, May/June 23.

\section{BYU ScholarsArchive Citation}

Archibald, James K.; Hall, Eric S.; Knutson, Charles D.; and Vawdrey, David K., "Enabling Remote Access to Personal Electronic Medical Records" (2003). Faculty Publications. 497.

https://scholarsarchive.byu.edu/facpub/497

This Peer-Reviewed Article is brought to you for free and open access by BYU ScholarsArchive. It has been accepted for inclusion in Faculty Publications by an authorized administrator of BYU ScholarsArchive. For more information, please contact ellen_amatangelo@byu.edu. 


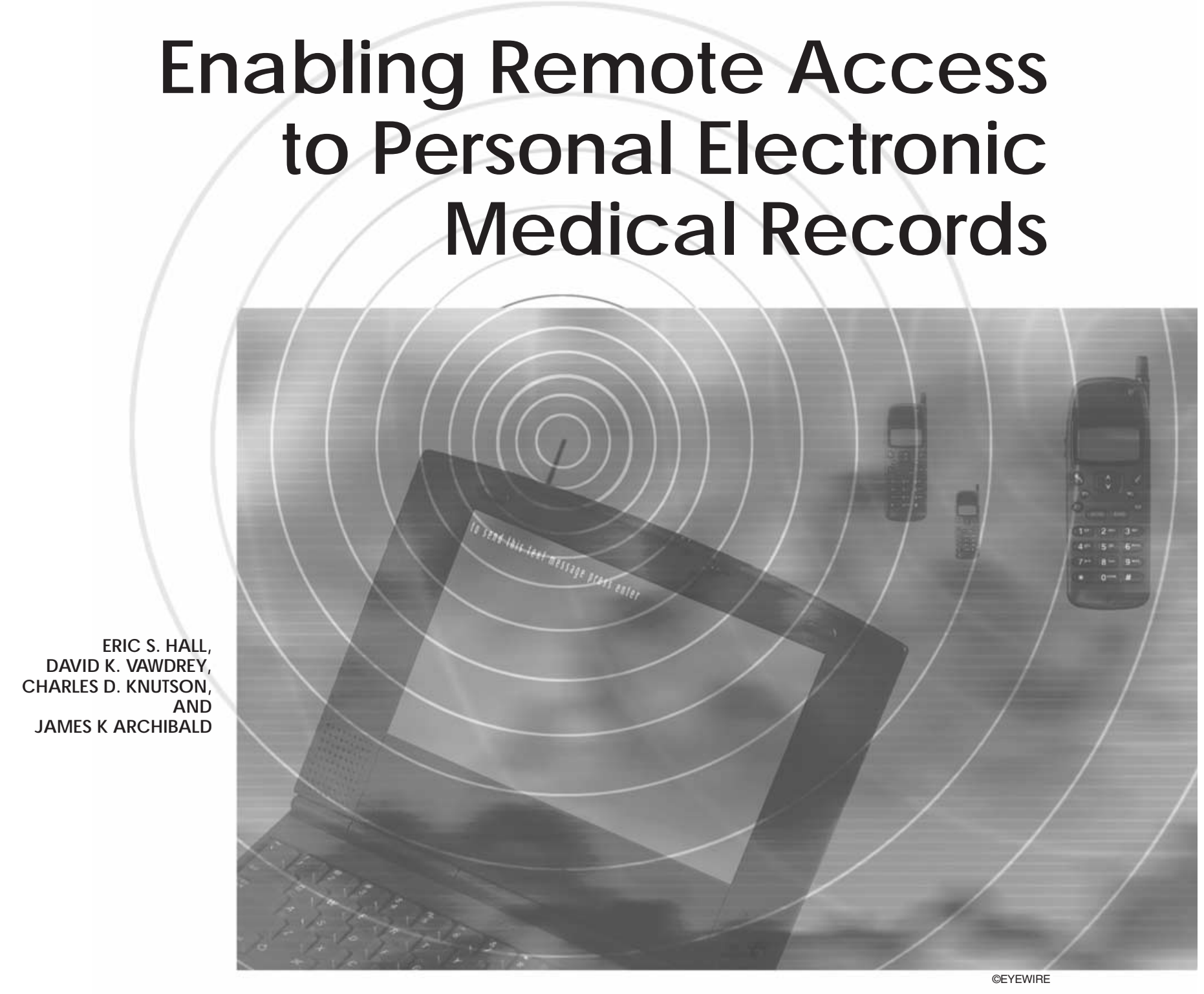

The Poket Doktor Is a Wireless

Personal Healthcare System

That Can Obtain Accurate

Patient Medical Information in

Situations Where It May Not

Otherwise Be Available

illions of people suffer from medical conditions that should be made known to healthcare practitioners prior to treatment. Paramedics and emergency room doctors cannot provide optimal care without sufficient knowledge of a patient's medical history. Lacking patient information such as allergies, current prescriptions, and preexisting conditions, medical professionals are often forced to either delay treatment or rely on instincts. Medical mistakes in situations like these kill thousands of people and cost an estimated US\$37 billion each year in the United States [1].

With the advent of electronic medical records (EMRs), patient information can be stored in computer databases at hospitals and doctor's offices [2], [3]. Unfortunately, timely access to this information is often impossible, either because 1) the infrastructure required to communicate with the records database is not available [4], or 2) medical practitioners are not able to identify the patient to the infrastructure. These situations are especially prevalent in emergency medicine, when the difference between life and death is sometimes measured in minutes and seconds.

Recent advances in handheld mobile computers and wireless data communication provide some of the tools necessary to help 
overcome these challenges. Capitalizing on these advances, the Poket Doktor project at Brigham Young University allows individuals to store personal medical information using portable electronic devices. This article discusses the first phase of the project consisting of the design and prototype development of a Bluetooth-enabled smart card that stores EMR data as well as the creation of a handheld computer application that reads the card and displays patient information. It summarizes recent relevant work, provides an overview of the Poket Doktor system architecture, describes the details of the smart card and application software prototypes, and discusses testing requirements and future work.

\section{Related Work}

To facilitate the growth of EMR systems, several groups are creating standards for the formatting and storage of electronic patient data. Since its formation more than 15 years ago, Health Level Seven (HL7) has sought to provide a comprehensive framework to manage the exchange, integration, sharing, and retrieval of electronic health information [5]. Standards developed by organizations within the medical informatics community (such as DICOM, LOINC, and SNOMED) address similar concerns in medical imaging and treatment classification.

Other researchers are involved in specific projects focused on storing and exchanging EMR data. The Ward-in-Hand project is improving hospital care by supplying medical practitioners with mobile computers, giving them wireless access to patient records on a centralized server [6]. Other researchers have examined the potential of extending current electronic medical information infrastructures (EMIIs) to allow access to hospital resources from ambulances equipped with cellular and other wireless communication technologies [7], [8].
Despite significant progress in standardizing medical record data and sharing information via wired and wireless EMIIs, at least two fundamental problems remain. First, it may be impossible for emergency workers to access an EMII, particularly if they are out of range or the infrastructure is damaged (as in a natural disaster). Second, if the patient is unconscious or incoherent, medical professionals may not be able to identify the patient to the infrastructure.

To overcome these problems, some groups are examining systems where EMR data is kept in possession of the patient [9]. In this patient-centered model, medical practitioners can access critical patient information even if they cannot connect with or identify the patient to the EMII. One example of this approach is the computerized personal information carrier (PIC) being developed by the U.S. Armed Forces [10]. Maintaining the same form factor as traditional military dog tags, the PIC can store a soldier's identification information and up to $8 \mathrm{MB}$ of audio, video, and text-based EMR data.

While the PIC concept is well suited for military applications, expanding this idea to civilian markets causes serious concerns in locating and identifying patient devices, maintaining privacy and confidentiality, and establishing standards to guarantee interoperability [11]. For example, when treating an unconscious patient, medical workers typically do not have the option of searching the patient's personal belongings to locate a device containing EMR data. Taking advantage of recent technological advances, we have designed a broad, flexible architecture called the Poket Doktor System that addresses these concerns.

\section{Poket Doktor System Arc hitecture}

The Poket Doktor System allows a user to store EMR data on a personal electronic device and wirelessly communicate this data to medical professionals when treatment is required [12].

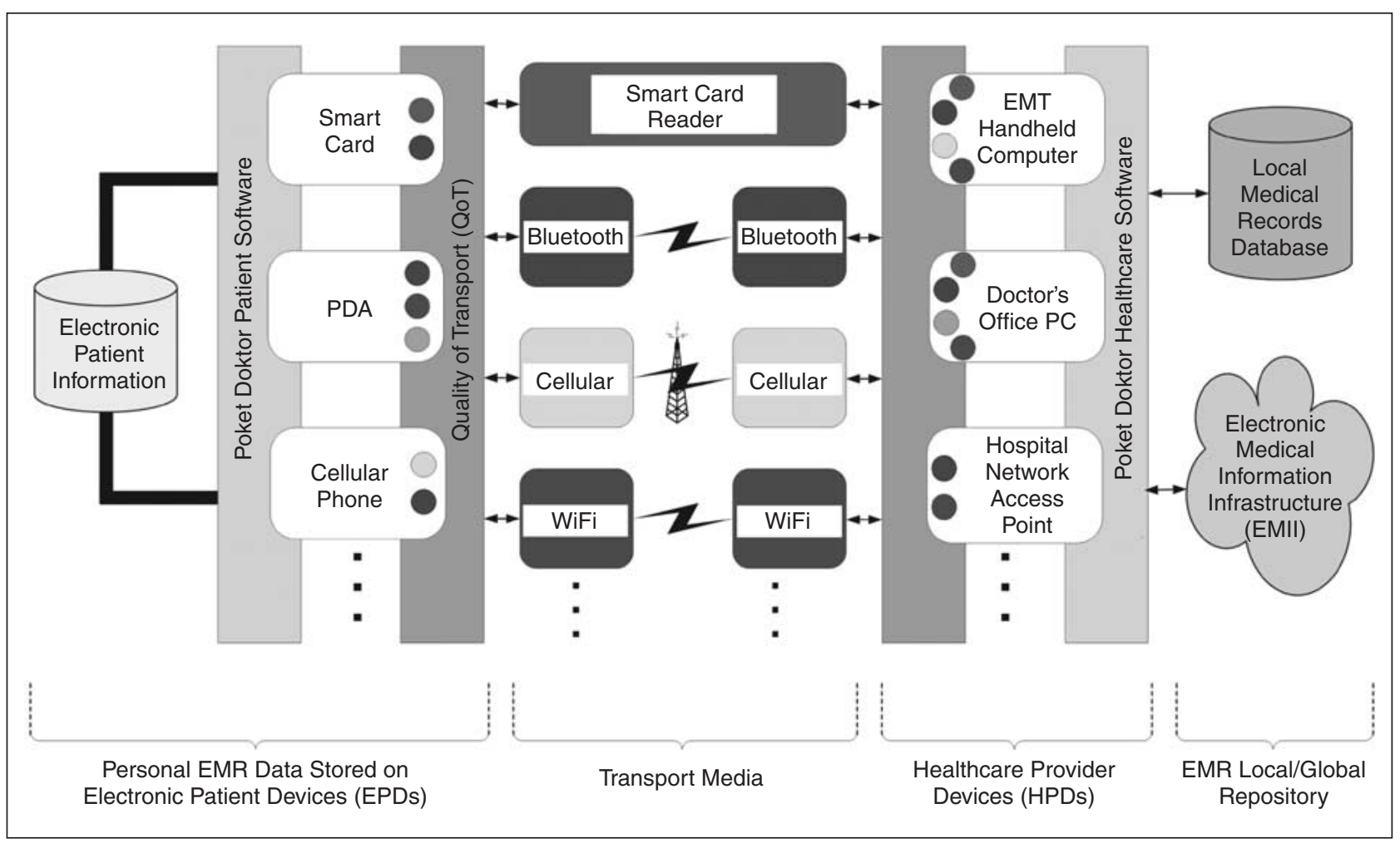

Fig. 1. Poket Doktor system a rc hitec ture. 
Perhaps most noteworthy, in a disaster or other emergency situation, medical workers can even access a patient's Poket Doktor device without physical access to the device itself. This functionality makes the system extremely beneficial when little or no patient medical information is otherwise available.

Two specific advances in technology facilitate the notion of storing personal EMR data in electronic devices:

> Proliferation of Mobile Computing Devices. Mobile computing devices (such as smart cards, cellular phones, PDAs, and pagers) have become smaller, more powerful, and more capable of storing information. The Poket Doktor System architecture accommodates multiple platforms, taking advantage of their respective capabilities.

> Ubiquitous Wireless Data Communications Technologies. Wireless technologies like Bluetooth, 802.11 (WiFi), Ultrawideband (UWB), and cellular communication are becoming increasingly widespread, and emerging solutions are well suited for use in mobile devices. Since it is unlikely that a single wireless transport will become predominant, contending with multiple transports will be a fact of life for some time to come. The Poket Doktor System employs a mechanism called quality of transport (QoT) to allow dynamic and transparent switching between available transport protocols.

The Poket Doktor System architecture is designed to allow an individual to carry personal electronic medical information on a wireless handheld device such as a cell phone, PDA, or enhanced smart card (see Figure 1). Medical workers can obtain this information wirelessly using handheld devices, desktop computers, network access points, etc. In this way, patients become an active component of the medical information infrastructure, facilitating better delivery of medical care.

Key components of the Poket Doktor System include the following.

> An electronic patient device (EPD) based on a handheld computing platform such as a smart card, PDA, cellular telephone, etc. EPDs include a patient's critical medical information, system and application software, and one or more wireless transport mechanisms for data exchange.

> A healthcare provider device (HPD), which may be a PDA, a desktop PC, or a network access point in a hospital. Healthcare practitioners use HPDs to communicate wirelessly with EPDs and also to access existing EMIIs. HPDs contain system and application software that provide this functionality.

> Quality of transport (QoT), an enabling technology that permits a device to dynamically and transparently change underlying data transport protocols without disturbing the communication session [13]. QoT provides a mechanism for maintaining a connection under changing environmental conditions as well as for optimizing communication based on desired specifications.

The Poket Doktor System architecture takes advantage of popular, well-documented standards developed within the medical informatics and wireless data communications communities. For example, using HL7 and eXtensible Markup Language (XML) formatting in data storage helps to ensure compatibility with existing EMR databases. Flexibility within the system to include security measures such as strong encryption and user-regulated access helps to guarantee confidentiality and compliance with privacy laws. Using established wireless data communication technologies reduces problems with medical device interference and facilitates interoperability. In short, the architecture is comprehensive enough to satisfy information storage and communications requirements, yet it remains simple enough to meet the needs of patients and healthcare providers.

\section{Poket Doktor Preliminary Prototype}

The following subsections describe the first phase of Poket Doktor System development, consisting of the design and implementation of a smart-card-based EPD and the development of application software for an HPD.

\section{Smart-Card EPD}

The smart-card EPD is equipped with solid-state memory and short-range wireless communication capabilities, allowing it to store and transmit EMR data. The functional requirements for the device with respect to smart-card platform, communication capabilities, and power efficiency are discussed below.

\section{Smart-Card Platform}

Traditional smart cards provide sufficient storage capacity and security features to make them an excellent solution for storing EMR information. Smart cards offer a form factor similar to credit cards and are capable of storing several megabytes of relevant medical data. Some manufacturers are adding microcontrollers to smart cards to increase security and to provide other functionality (including I/O capabilities). Figure 2 shows the block diagram for the Poket Doktor Smart Card EPD platform. It consists of a microcontroller-based smart card with a flat lithium-manganese dioxide battery and a wireless communications subsystem comprising a Bluetooth radio and a radio frequency identification (RFID) module. This design provides adequate processing power, storage capacity, and communication capabilities to fit the needs of the Poket Doktor EPD model.

\section{Wireless Communic a tion Capability}

Bluetooth wireless technology offers several features that make it an excellent medium for transmitting EMR data stored on a smart card. As an emerging standard in wireless data communications, Bluetooth provides robust, me-

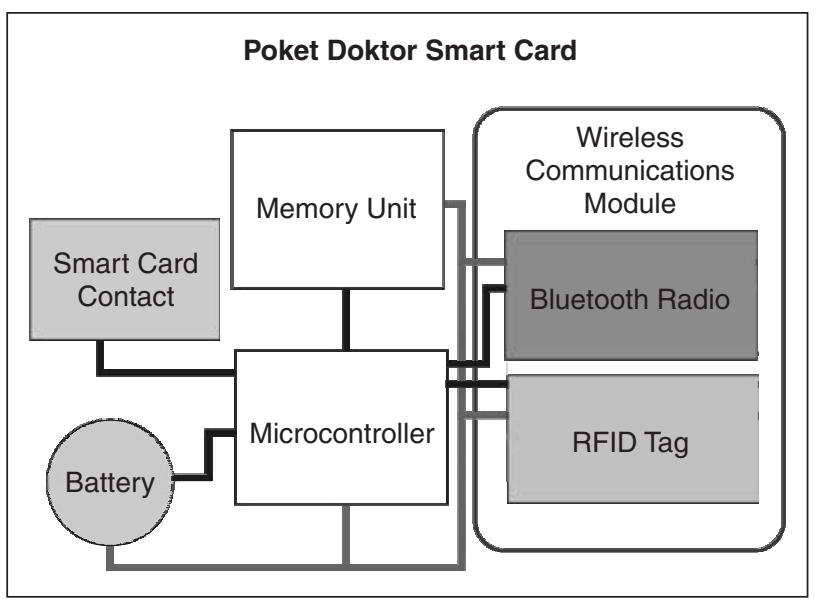

Fig. 2. Poket Doktor Smart Card block diagram. 
dium-bandwidth communication within a 10-100 m range. Low cost, minimal power consumption, and a small physical footprint make Bluetooth well suited for integration into embedded devices.

One limitation associated with Bluetooth is a lengthy discovery process. In some cases the standard 10.24-s delay in device discovery may be too long to allow timely and efficient communication between mobile devices. To overcome this problem we have developed a process called Rendez-Blue, which leverages the strengths of RFID technology to speed up the Bluetooth connection establishment process. (The second purpose of Rendez-Blue - reducing power consumption-is discussed in the next subsection.) RFID is an automatic data capture system that uses electromagnetic or inductive coupling to transmit a small amount of data from a uniquely programmed transponder, called an RF tag, to a transceiver or reader. In Rendez-Blue, RFID is used to communicate the EPD Bluetooth radio's hardware address to the querying device-the HPD containing the RFID transceiver. Receiving the Bluetooth address via RFID allows the HPD to bypass the standard Bluetooth inquiry process and proceed directly to connection establishment. Empirical testing has shown that this aspect of the Rendez-Blue process results in a four-tosix-fold reduction in device discovery time [14].

Another problem alleviated by Rendez-Blue is the difficulty that would arise if an emergency occurred in a crowded area where many people were carrying Poket Doktor Smart Card EPDs. In this situation, it is not efficient for medical workers to wirelessly scan a large area seeking to locate the Poket Doktor Smart Card belonging to the patient they are at-

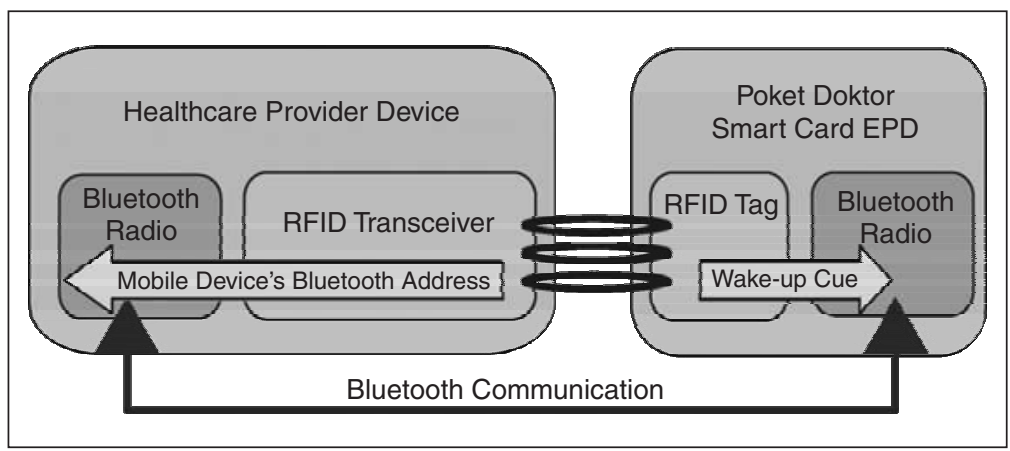

Fig. 3. Rendez-Blue process used to activate the smart card and establish a Bluetooth connection.

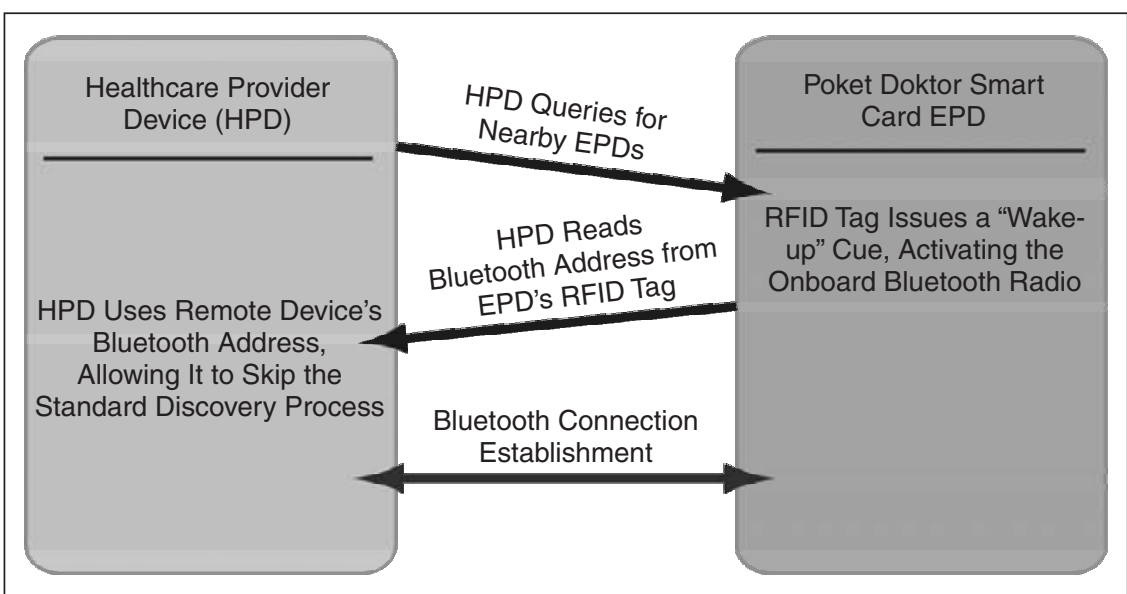

Fig. 4. Rendez-Blue connection establishment procedure. tempting to treat. Rendez-Blue allows medical professionals to effectively vary the search radius, so they can discover and connect with smart-card EPDs at distances ranging from a few inches to several meters.

\section{Power Conservation Module}

In addition to enhancing the mechanism for locating and connecting to Poket Doktor smart cards, Rendez-Blue plays a major role in power conservation. In portable electronic devices, battery life fundamentally determines the extent of the device's mobility [15]. Because increased sophistication of mobile systems implies a commensurate increase in power requirements, the design and implementation of power-conserving schemes is crucial [16]. In devices such as the Poket Doktor Smart Card EPD that have strict size and weight limitations, this principle is especially relevant.

To provide maximum power savings, the Bluetooth module on the Poket Doktor Smart Card should respond to an inquiry and establish a connection only when it is communicating with an HPD. Power should not be wasted responding to discovery inquiries from cell phones, MP3 players, or other Bluetooth-enabled devices. To prevent this from happening, the Bluetooth radio cannot remain in a powered state-it should be shut down whenever possible. In the smart-card EPD, the Bluetooth module is essentially "asleep" when it is not needed. To activate the Bluetooth radio when communication is desired, we employ a technique referred to as node rendezvous [17], where a low-power device (the RFID $\operatorname{tag}$ ) is externally triggered to send a "wake-up" cue [14]. This process is illustrated in Figure 3.

RFID technology is an ideal mechanism for facilitating the power-saving aspect of Rendez-Blue because the passive RFID tag used in the Poket Doktor Smart Card is lightweight, inexpensive, and durable [18]. Even more important, the RFID tag is "passive," requiring no onboard power supply. Operating power for the tag is derived from the energy pulse sent from the HPD's RFID transceiver. The virtually unlimited operational lifetime of RF tags makes them very attractive for use in small mobile devices like the Poket Doktor Smart Card EPD.

Figure 4 demonstrates the process through which an HPD discovers and establishes a Bluetooth connection with a Poket Doktor Smart Card using Rendez-Blue. First, a medical professional uses an HPD to query for nearby smart-card EPDs. The RFID tag on the smart card receives this query and then issues a "wake-up" cue, activating the Bluetooth radio. Simultaneously, the data stored in the RFID tag, namely the hardware address of the Bluetooth radio, is read via RFID by the medical worker's HPD. Once the HPD obtains the EPD's Bluetooth address, Bluetooth connection establishment can proceed normally. Employing Rendez-Blue in the Poket Doktor System prevents the constant power drain of the Bluetooth radio 


\section{Perhaps most noteworthy, in a disaster \\ or other emergency situation, medical \\ workers can access a patient's \\ Poket Doktor device without physical \\ access to the device itself.}

when not in use and reduces device discovery delays when communication is desired.

\section{HPD Handheld Computer and Application Software}

Prototype development of application software has been completed, allowing desktop and handheld computers to access EMR data on Poket Doktor Smart Cards. The software architecture model is depicted in Figure 5.

The QoT component determines the most appropriate transport medium to use for a given information exchange scenario. For example, at a doctor's office, the patient's card can be inserted into a smart-card reader to synchronize recent EMR data. In contrast, a paramedic in an emergency situation might use the wireless capabilities offered by Bluetooth technology to locate the smart card, identify the patient, and gain access to critical information that will facilitate optimal treatment.

A variety of desktop and mobile computers are currently being used in the healthcare industry to access EMR informa- tion. The Poket Doktor System is designed to ensure compatibility with existing hardware and software systems. With this in mind, the application software easily integrates with existing desktop terminals at hospitals and doctor's offices. Computers at these locations can be easily outfitted with smart card readers to accommodate Poket Doktor Smart Cards. In mobile devices, Bluetooth is becoming increasingly pervasive, and it will provide flexibility to healthcare providers, allowing them to access Poket Doktor Smart Cards without a significant investment in new hardware.

In addition to providing platform flexibility, the application software is designed to meet the following standards:

> Speed: In an emergency, time is critical. Establishing a communication link, transferring data, and decrypting secure information must be timely and efficient.

$>$ Reliability: Software must be free of defects and allow hardware to function under adverse conditions.

- Usability: Medical practitioners and emergency workers are often required to perform several tasks at once. The

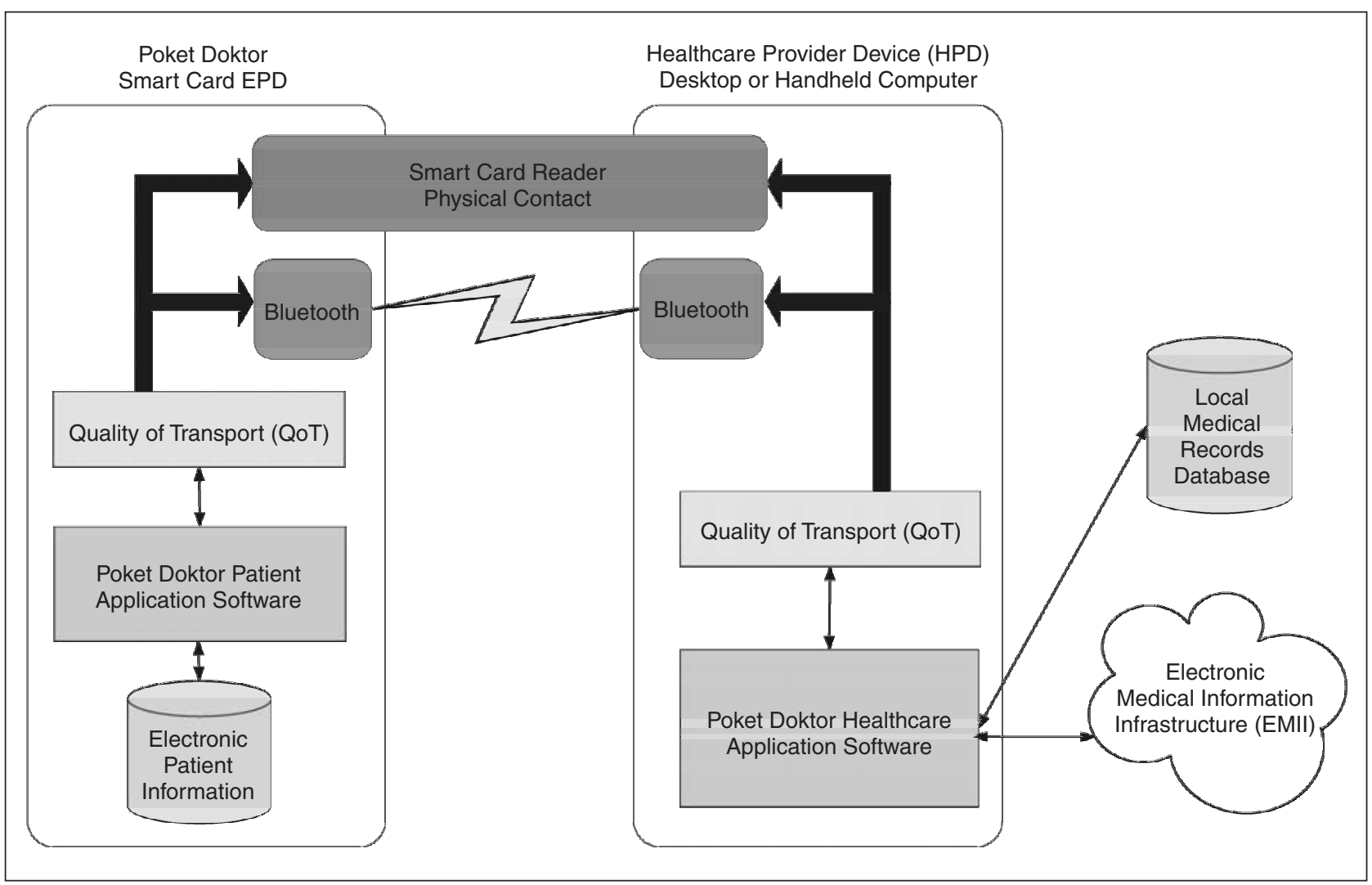

Fig. 5. Software arc hitec ture model for Poket Doktor prototype. 
user-interface of the application software must be easy to operate so the medical worker can focus on the patient.

- Security: Medical information is highly confidential. All sensitive data must be secure from unauthorized access. Initial prototypes include security features such as secure key-exchange and strong data encryption as well as multiple levels of access to information via password protection.

\section{The Hand held Devic e User Interface}

To meet these requirements, graphical user interfaces (GUIs) allow the user to execute commands and view information in a user-friendly environment. Simple on-screen buttons allow a medical worker to easily perform functions such as discover-

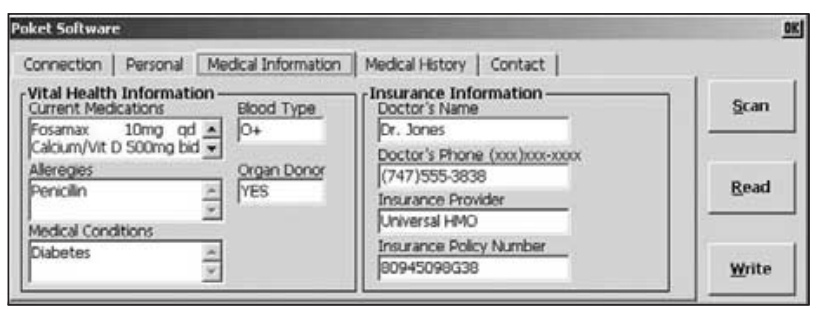

Fig. 6. Screen capture of Poket Doktor software running on handheld computer.

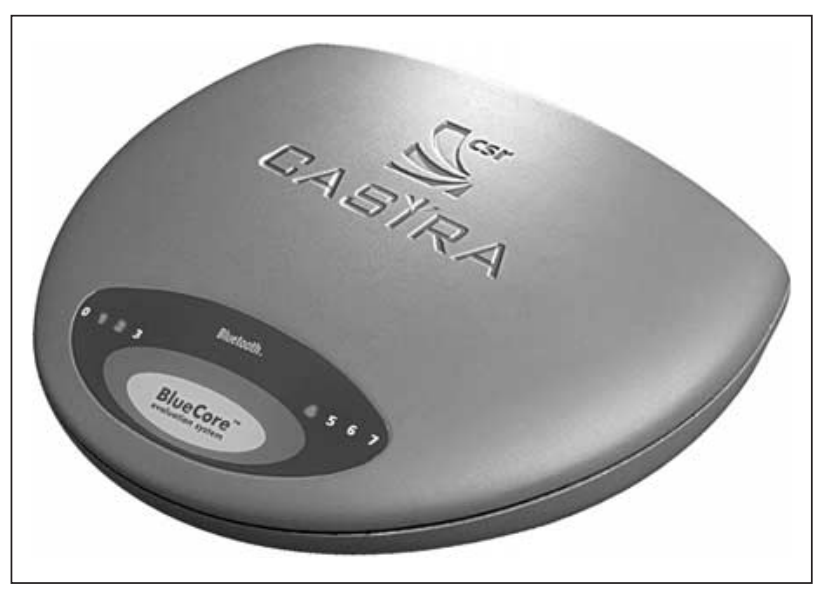

Fig. 7. Casira Bluetooth development kit used to create Poket Doktor Smart Card prototype.

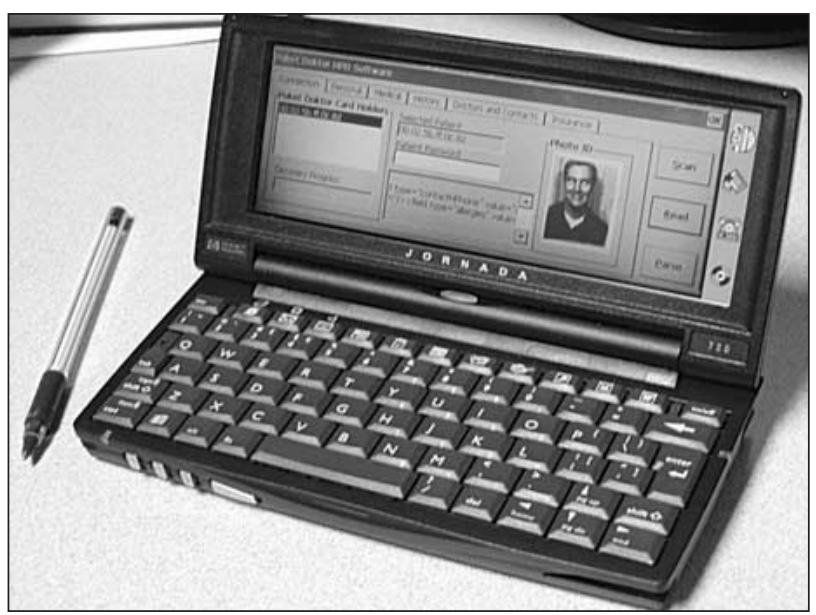

Fig. 8. Handheld computer HPD based on the HP J omada 720 running Poket Doktor a p lic ation software. ing patient smart cards and retrieving EMR data (see Figure 6). The GUI also provides the capability of displaying a photograph of the smart card owner for identification purposes.

\section{Data Formatting}

To allow standardization and efficiency in retrieving and parsing data, the Poket Doktor application stores medical records using XML. XML is widely used within the HL7 standards for use in exchanging clinical information. Storing data is this format is beneficial because it takes advantage of existing standards and provides consistency and structure in the way data is exchanged.

\section{Security}

The Poket Doktor System provides a number of security features to protect against unauthorized access to a patient's sensitive healthcare information. One of the primary vulnerabilities inherent in wireless communication is the threat of eavesdropping. Experts suggest that this is a significant danger, even if two devices are communicating only at short range [19]. Bluetooth provides a limited number of low-level security measures to prevent eavesdropping, and more advanced security procedures (e.g., public keys, certificates) can be implemented within the Poket Doktor System to provide greater protection. The software prototype supports secure key-exchange methods and strong conventional encryption algorithms such as 3DES and AES. This allows sensitive medical information to be stored and communicated securely, ensuring that unauthorized Bluetooth devices cannot access confidential information [20].

In a medical emergency situation where a patient is unresponsive, it is important that authorized medical workers are able to obtain information critical to administering proper treatment. At the same time however, patients may not want to allow unlimited access to personal data without consent. Therefore, the Poket Doktor Smart Card is designed to allow the owner to selectively restrict access to certain data stored on the card (such as insurance information or social security number). When a card is initialized at a hospital or doctor's office, the patient may choose a password and specify certain data fields as accessible and others as restricted. Access to restricted fields is granted only when the card owner supplies the correct password. Selected blocking of information enables personalized medical care without sacrificing the patient's right to privacy.

\section{Current and Future Work}

The first phase of Poket Doktor development provides a proof-of-concept prototype to demonstrate the functionality and usability of the architecture. The smart-card EPD prototype achieves all basic goals for functionality, and development is continuing in order to meet size and power constraints. The current implementation was developed using Casira $^{\text {TM }}$ Bluetooth Development Kits contributed by Cambridge Silicon Radio (see Figure 7). The kit features a BlueCore Single Chip Bluetooth solution with a 6 x $6 \mathrm{~mm}$ footprint, an internal microcontroller, and multiple I/O interfaces. Poket Doktor Smart Cards based on the BlueCore system should be relatively inexpensive to manufacture; current estimates suggest a retail cost of around US $\$ 25$.

The handheld computer HPD platform is based on the HP Jornada 720 augmented with Bluetooth and RFID communi- 


\section{When a card is initialized at a hospital or doctor's office, the patient may choose a password and specify certain data fields as ac cessible and others as restricted.}

cations capabilities. The application software is written in Embedded Visual $\mathrm{C}++$ and can be easily ported to devices running the PocketPC operating system.

Future development of the Poket Doktor will transform the current prototypes into working products that will greatly benefit patients and healthcare practitioners. Additional development, verification, and validation will facilitate field testing within intended application environments (such as accident scenes and hospital emergency rooms). Future development phases will also address expanding the architecture to include other EPD and HPD platforms, incorporating QoT technology to manage wireless data communication sessions, and interfacing and synchronizing Poket Doktor EPD data with existing records repositories.

\section{Conclusion}

The Poket Doktor system is designed to provide a flexible, scalable method of storing and communicating critical EMR information using personal handheld electronic devices. The first phase of development has succeeded in:

> designing the architecture for a wireless, power-efficient smart card to store and communicate medical information

> incorporating Bluetooth wireless technology with RFID wakeup on the smart card to enable a fast wireless connection to a healthcare provider's device; and

> selecting a platform and creating application software for a handheld computing device used by healthcare providers.

The Poket Doktor system assists medical personnel in obtaining accurate patient medical information in situations where it may not otherwise be available. In this manner, Poket Doktor technology will improve the quality of care delivered in emergency situations.

Eric S. Hall is a graduate researcher in the Mobile Computing Laboratory at the Brigham Young University's Computer Science Department. His research interests focus on mobile computing and digital design.

David K. Vawdrey is a graduate researcher in the Mobile Computing Laboratory at the Brigham Young University's Computer Science Department. His research interests include embedded systems design and short-range wireless data communications in the context of medical records exchange.

Charles D. Knutson is an assistant professor of computer science at Brigham Young University and director of the Mobile Computing Laboratory. He holds a Ph.D. in computer science from Oregon State University.

James K Archibald is an associate professor of electrical and computer engineering at Brigham Young University. He holds a Ph.D. in computer science from the University of Washington. His research interests are in embedded and robotic systems.

Address for Correspondence: David Vawdrey, Mobile Computing Lab, Brigham Young University, 2204 TMCB, Provo, UT 84602 USA. Tel: +1 801422 9123. E-mail: dkv@email. byu.edu.

\section{References}

[1] Institute of Medicine, To Err is Human: Building a Better Health Care System. Reading, MA: Addison-Wesley, 1998.

[2] R.S. Dick, E.B. Steen, and D.B. Detmer, The Computer-Based Patient Record: An Essential Technology for Health Care, rev. ed. Washington, DC: National Academy Press, 1997.

[3] J. Van der Lei, P.W. Moorman, and M.A. Musen, "Electronic patient records in medical practice," Meth. Inf. Med., vol. 38, no. 4-5, pp. 287-288, 2000.

[4] K.D. Mandl, I.S. Kohane, and A.M. Brandt, "Electronic patient-physician communication: Problems and promise," Ann. Int. Med., vol. 129, no. 6, pp. 495-500, 1998. [5] Health Level Seven. Available: http://www.hl7.org

[6] M. Ancona, E. Coscia, G. Dodero, M. Earney, V. Gianuzzi, F. Minuto, and S. Virtuoso, "Ward-In-Hand: Wireless access to clinical records for mobile healthcare professionals," TEHRE 2001 m-Health Conf., 1st Annu. Conf. Mobile Wireless Healthcare Applications, London, U.K., Nov. 11-14, 2001.

[7] S. Morton and O. Bukhres, "Utilizing mobile computing in the Wishard Memorial Hospital ambulatory service," in Proc. ACM Symp. Appl. Comp., San Jose, CA, Feb. 28-Mar. 2, 1997, pp. 287-294.

[8] S. Pavlopoulos, E. Kyriacou, A. Berler, S. Dembeyiotis, and D. Koutsouris, "A novel emergency telemedicine system based on wireless communication technology-AMBULANCE," IEEE Trans. Inform. Technol. Biomed., vol. 2, pp. 261-267, 1998.

[9] P. Szolovits, J. Doyle, J. William, I. Kohane, and S. Pauker, "Guardian Angel: Patient-centered health information systems," MIT Lab. Comput. Sci., Cambridge, MA, Tech. Rep. TR-604, May 1994.

[10] P. Gunby, "Test of a new medical dog tag with civilian potential," JAMA, vol. 279, no. 2, pp. 99-100, 1998.

[11] T. Huston, "Security issues for implementation of e-medical records," Comm. ACM, vol. 44, no. 9, pp. 89-94, 2001.

[12] D.K. Vawdrey, E.S. Hall, C.D. Knutson, and J.K. Archibald, "A self-adapting, healthcare information infrastructure using mobile computing devices," in 5th Int. Workshop Enterprise Networking and Computing Healthcare Industry (HealthCom 2003), Santa Monica, CA, June 6-7, 2003.

[13] R.W. Woodings, "Quality of Transport (QoT): Dynamic transport switching in wireless personal area networks," M.S. thesis, Dept. Comp. Sci., Brigham Young Univ., Provo, UT, Dec. 2002.

[14] E.S. Hall, D.K. Vawdrey, and C.D. Knutson, "RF Rendez-Blue: Reducing power and inquiry costs in Bluetooth-enabled mobile systems," in Proc. 11 th IEEE Int. Conf. Comput. Communications and Networks (ICCCN), Miami, FL, Oct. 2002, pp. 640-645.

[15] D. Panigrhia, C. Chiasserini, S. Dey, R. Rao, A. Raghunathan, and K. Lahiri, "Battery life estimation of mobile embedded systems," in Proc. 14th Int. Conf. VLSI Design, Bangalore, India, Jan. 7-11, 2001, pp. 57-63.

[16] F. Koushanfar, V. Prabhu, M. Potkonjak, and J.M. Rabaey, "Processors for mobile applications," in Proc. 2000 Int. Conf. Comput. Design (ICCD), Austin, TX, Oct. 25-27, 2000, pp. 603-608.

[17] T. Todd, F. Bennett, and A. Jones, "Low power rendezvous in embedded wireless networks," 1st Annu. IEEE Workshop Mobile Ad Hoc Networking and Computing (MobiHOC), Boston, MA, Aug. 11, 2000.

[18] M. Nosovic and T. Todd, "Low power rendezvous and RFID wakeup for embedded wireless networks," in Proc. 15th Annu. IEEE Computer Communications Workshop (CCW), Captiva Island, FL, Oct. 15-18, 2000.

[19] J.C. Haartsen, "The Bluetooth radio system," IEEE Pers. Commun., vol. 7, pp. 28-36, 2000.

[20] T.C. Rindfleisch, "Privacy, information technology, and health care," Comm. ACM, vol. 40, no. 8, pp. 92-100, 1997. 